\title{
Curcumin and Rutin Down-regulate Cyclooxygenase-2 and Reduce Tumor-associated Inflammation in HPV16-Transgenic Mice
}

\author{
MAGDA S.S. MOUTINHO ${ }^{1}$, SOFIA ARAGÃO ${ }^{1}$, DIOGO CARMO ${ }^{2}$, FÁTIMA CASACA $^{2}$, \\ SANDRA SILVA ${ }^{2}$, JOANA RIBEIRO ${ }^{3}$, HUGO SOUSA ${ }^{3}$, ISABEL PIRES ${ }^{4,5}$, FELISBINA QUEIROGA ${ }^{1,4,6}$, \\ BRUNO COLAÇO ${ }^{1,7}$, RUI MEDEIROS ${ }^{3,8,9,10}$, PAULA A. OLIVEIRA ${ }^{1,4}$, CARLOS LOPES $^{2}$, \\ MARGARIDA M.S.M. BASTOS ${ }^{11}$ and RUI M. GIL DA COSTA ${ }^{1,3,11}$ \\ ${ }^{1}$ Center for the Research and Technology of Agro-Environmental and Biological Sciences, \\ ${ }^{4}$ Department of Veterinary Sciences, ${ }^{5}$ Animal and Veterinary Research Center-Department of Veterinary Sciences, \\ ${ }^{7}$ Zootechnics Department, University of Trás-os-Montes and Alto Douro, Vila Real, Portugal; \\ ${ }^{2}$ Botelho Moniz Análises Clínicas, Porto, Portugal; \\ ${ }^{3}$ Molecular Oncology and Viral Pathology Group, IPO-Porto Research Center, \\ Portuguese Institute of Oncology of Porto, Porto, Portugal; \\ ${ }^{6}$ Center for the Study of Animal Science, ${ }^{11}$ Laboratory for Process Engineering Environment Biotechnology and \\ Energy (LEPABE) Chemical Engineering Department, Faculty of Engineering, and \\ ${ }^{8}$ Faculty of Medicine, University of Porto, Porto, Portugal; \\ ${ }^{9}$ Center for Biomedical Research, Faculty of Health Sciences, Fernando Pessoa University, Porto, Portugal; \\ ${ }^{10}$ Portuguese League Against Cancer, Research Department (NRNorte), Porto, Portugal
}

\begin{abstract}
Aim: Cyclo-oxygenase-2 (COX2) plays a prominent role in carcinogenesis. This study addresses the effects of two nutraceutical compounds on the expression of COX2 and tumor-associated inflammation in human papillomavirus type 16 (HPV16)-transgenic mice. Materials and Methods: Sixweek-old $F V B / n$ mice were supplemented with rutin or curcumin for 24 weeks: HPV16 ${ }^{-/-}$no treatment, $n=12$; $H P V 16^{+/-}$no treatment, $n=13 ; H P V 16^{+/-}$rutin, $n=12$; $\mathrm{HPVl6}^{+/-}$curcumin, $n=13$. HPV16-induced skin lesions and their inflammatory infiltrates were studied histologically. COX2 expression was assessed immunohistochemically. Results: Rutin reduced COX2 expression in the dermis (immunostaining score 7.83 versus 11.25 in untreated HPV16-transgenic mice) and epidermis (4.5 versus 10.0). Curcumin led to dermal and epidermal scores of 10.5 and 4.5. Both compounds reduced leukocytic infiltration, but neither prevented epidermal dysplasia. Conclusion: COX2 expression in HPV16-induced
\end{abstract}

Correspondence to: Magda Moutinho, Center for the Research and Technology of Agro-Environmental and Biological Sciences, University of Trás-os-Montes and Alto Douro, Quinta de Prados, Vila Real, Portugal. Tel: +351 259350000, Fax: +351 259350480, e-mail: magdamoutinho@hotmail.com

Key Words: HPV, mouse model, inflammation, nutraceutic, diet. lesions may be modulated by nutraceuticals, reducing tumorassociated inflammation. However, this was not sufficient to block carcinogenesis, calling for additional studies focused on combination therapies.

Infection by high-risk human papillomavirus (HPV) types, most prominently types 16 and 18 (HPV16 and HPV18) is associated with cancer of the uterine cervix and of other anatomic locations, such as the anus and the oropharynx (1). The development of HPV-associated pre-neoplastic and neoplastic lesions is associated with significant inflammatory phenomena (2), which promote stromal remodeling, angiogenesis and metastasis, and are coordinated by nuclear factor kappa B (NF-kB), as recently reviewed (3). Cyclooxygenase-2 (COX2), a key player in cancer-associated inflammation, is up-regulated by HPV16 E5, E6 and E7 oncoproteins, though NF-kB and the epithelial growth factor receptor (EGFR)-Ras-mitogen-activated protein kinase (MAPK) pathways $(4,5)$. In cervical lesions, COX2 upregulation modulates the immune response, promoting immunotolerance and lesion persistence (6). In line with these findings, celecoxib, a selective COX2 inhibitor, was recently reported to enhance the degranulation of $\mathrm{CD}^{+} \mathrm{T}$-cells in skin lesions induced by HPV16 oncogenes in transgenic (K14HPV16) mice (7). In the same study, celecoxib also reduced the incidence of cutaneous dysplastic lesions. This line of 
evidence suggests that COX2 may be a useful therapeutic target in HPV-induced cancer. Other anti-inflammatory substances have been tested against HPV-induced lesions, with different results. Among these are dietary polyphenols, most prominently curcumin $(8,9)$. Curcumin and other polyphenols such as quercetin (10) and resveratrol (11) inhibit NF-kB pathway signaling, down-regulating some of its downstream effectors, such as COX2. Recent observations showed that long-term oral administration of rutin (a quercetin glycoside) and curcumin ameliorated systemic inflammation and cachexia in K14-HPV16 mice (12). Considering these previous findings, and the role of COX2 in cancer induced by HPV, it has become essential to evaluate the expression of COX2 in the viral lesions of those animals. Furthermore, this study also describes how curcumin and rutin modulate COX2 expression, and their impact on tumor-associated inflammation.

\section{Materials and Methods}

Animals. K14-HPV16 mice on a FVB/n background (13), were generously donated by Drs. Jeffrey Arbeit and Douglas Hanahan, from the University of California, through the USA National Cancer Institute Mouse Repository. Animals were bred in the University of Trás-os-Montes and Alto Douro animal facility. Genotyping was performed as previously described $(14,15)$. Hemizygotic (HPV $16^{+/-}$) and wild-type (HPV16-/-) 6-week-old females were selected for use in the study. The study was authorized by the University de Trás-os-Montes e Alto Douro Ethics Committee (approval no. 10/2013) and the Portuguese Veterinary Directorate (approval no. 0421/000/000/2014).

Chemicals. Curcumin and rutin (Sigma-Aldrich, Saint-Louis, MA, USA) were dispersed in sunflower oil and coated onto diet pellets using an industrial mixer, to a final concentration of $2.0 \mathrm{mg} / \mathrm{g}$. The average daily doses of rutin and curcumin were $9.42 \pm 0.62 \mathrm{mg} / \mathrm{animal}$, or 413.0 and $422.4 \mathrm{mg} / \mathrm{kg}$, respectively. The final sunflower oil concentration was $3.0 \%(\mathrm{w} / \mathrm{w})$.

Experimental procedures. The animals were maintained in accordance with Portuguese (Decree-Law 113/2013, dated 7th August) and European (EU Directive 2010/63/EU) legislation, under controlled conditions of temperature $\left(23 \pm 2^{\circ} \mathrm{C}\right)$, light-dark cycle $(12$ $\mathrm{h}$ light $/ 12 \mathrm{~h}$ dark) and relative humidity $(50 \pm 10 \%)$, using hardwood bedding. Health checks were performed daily. As previously described (12), 50 mice were divided into four groups: 1 : HPV16 ${ }^{-/}$, $\mathrm{n}=12 ; 2: \mathrm{HPV}_{16} 6^{+/-}, \mathrm{n}=13 ; 3: \mathrm{HPV}_{16}^{+/-}, \mathrm{n}=12$; and $4: \mathrm{HPV} 16^{+/-}$, $\mathrm{n}=13$. Groups 3 and 4 were administered rutin, and curcumin, respectively, for 24 consecutive weeks. Groups 1 and 2 received a control diet (3.0\% sunflower oil). At 30 weeks old, the animals were sacrificed by intraperitoneal pentobarbital overdose followed by cardiac puncture and exsanguination.

Histological analysis. Chest skin samples were collected and fixed in $10 \%$ neutral-buffered formalin. Following histological processing, each skin sample was stained with hematoxylin and eosin and classified histologically as normal skin, epidermal hyperplasia epidermal dysplasia. Total infiltrating leukocytes and specific leukocytic populations (neutrophils, macrophages, lymphocytes, plasma cells, mast cells) were counted in high-power $(\times 400)$ fields and leukocyte counts are expressed as mean values \pm standard error to the mean.

Immunohistochemical analysis. Immunohistochemistry for COX2 (1:100; SP21; Transduction Laboratories, Lexington, KY, USA) was performed as previously described (16). Briefly, heat-induced epitope retrieval was performed in a microwave oven $(700 \mathrm{~W})$ for 20 minutes in a citrate buffer solution. Endogenous peroxidase activity was blocked with $3 \%$ hydrogen peroxide. Nonspecific staining was minimized by a 30 -minute incubation with normal rabbit serum (X 0902; DakoCytomation, Glostrup, Denmark). The primary antibody was incubated overnight $\left(4^{\circ} \mathrm{C}\right)$ in a humid chamber. Immunoreactivity was detected using a streptavidin-biotinperoxidase complex (TS-125-HR; Labvision Corporation, Freemont, CA, USA). Color development was performed using 3,3'diaminobenzidine tetrahydrochloride and the preparations were counterstained with Mayer's hematoxylin. For negative controls, sections were incubated with normal rabbit serum instead of the primary antibody. Macrophages on submandibular lymph nodes were used as internal positive controls. Immunostaining for COX2 was assessed on the dermis and epidermis according to its distribution and intensity. Concerning the distribution of positive cells, four staining patterns were observed: pattern 1, comprising cases where immunostaining was present in fewer than $25 \%$ of cells, pattern 2 with $26-50 \%$ of stained cells, pattern 3 with $51-75 \%$ of stained cells and pattern 4 with $76-100 \%$ of stained cells. The staining intensity was scored as light ( 1 point), moderate ( 2 points) or intense ( 3 points). The COX2 score represents the product of the pattern of positive tumor cells ( 1 to 4 points) and staining intensity ( 1 to 3 points) and ranged between 0 and 12 .

Statistics. The Shapiro-Wilk test rejected the hypothesis that group data were samples from a normal distribution. Scale transformations did not change the previous hypothesis rejection. The nonparametric Kruskal-Wallis test was applied, the significance level was set to 0.05 and the significance values were adjusted by the Bonferroni correction for multiple tests. All statistical analyses were carried out using SPSS 24 (IBM Corp., Armonk, NY, USA). Results were expressed as mean \pm standard deviation and mean rank, when appropriate.

\section{Results}

Cutaneous lesions and leukocytic infiltrates. No histological alterations were observed in control (group 1) animals. All treated and untreated transgenic mice (groups 2-4) showed varying degrees of epidermal hyperplasia and dysplasia (Figure 1), and there were no significant differences between groups, as previously described (12). HPV-transgenic mice showed increased numbers of tumor-associated leukocytes compared with wild-type animals (Table I). Rutin and, to a lesser extent, curcumin, reduced the leukocytic infiltration in HPV-induced lesions (Table I).

COX2 immunoexpression. The normal skin of wild-type mice did not express COX2, neither at the dermal nor epidermal level (Figure 2a). Untreated transgenic animals (group 2) showed 


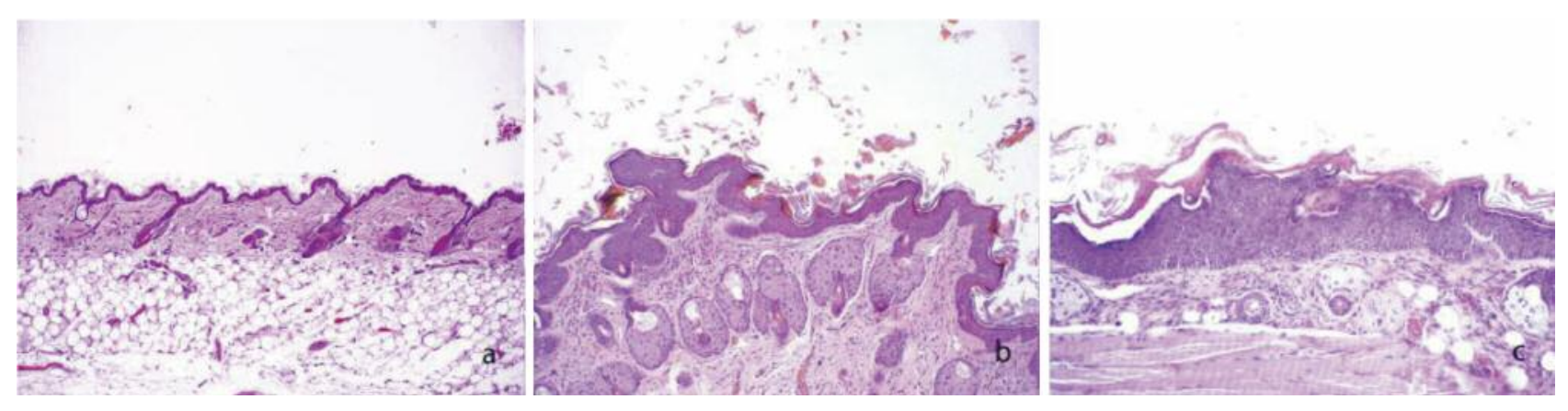

Figure 1. Histopathological changes induced by HPV16 in FVB/n mice, hematoxylin-eosin staining. a: Normal skin histology (group 1 animal), $\times$ 40. b: Epidermal hyperplasia and papillomatosis (group 2 animal), $\times 100$. : : Epidermal dysplasia (group 2 animal), $\times 100$.
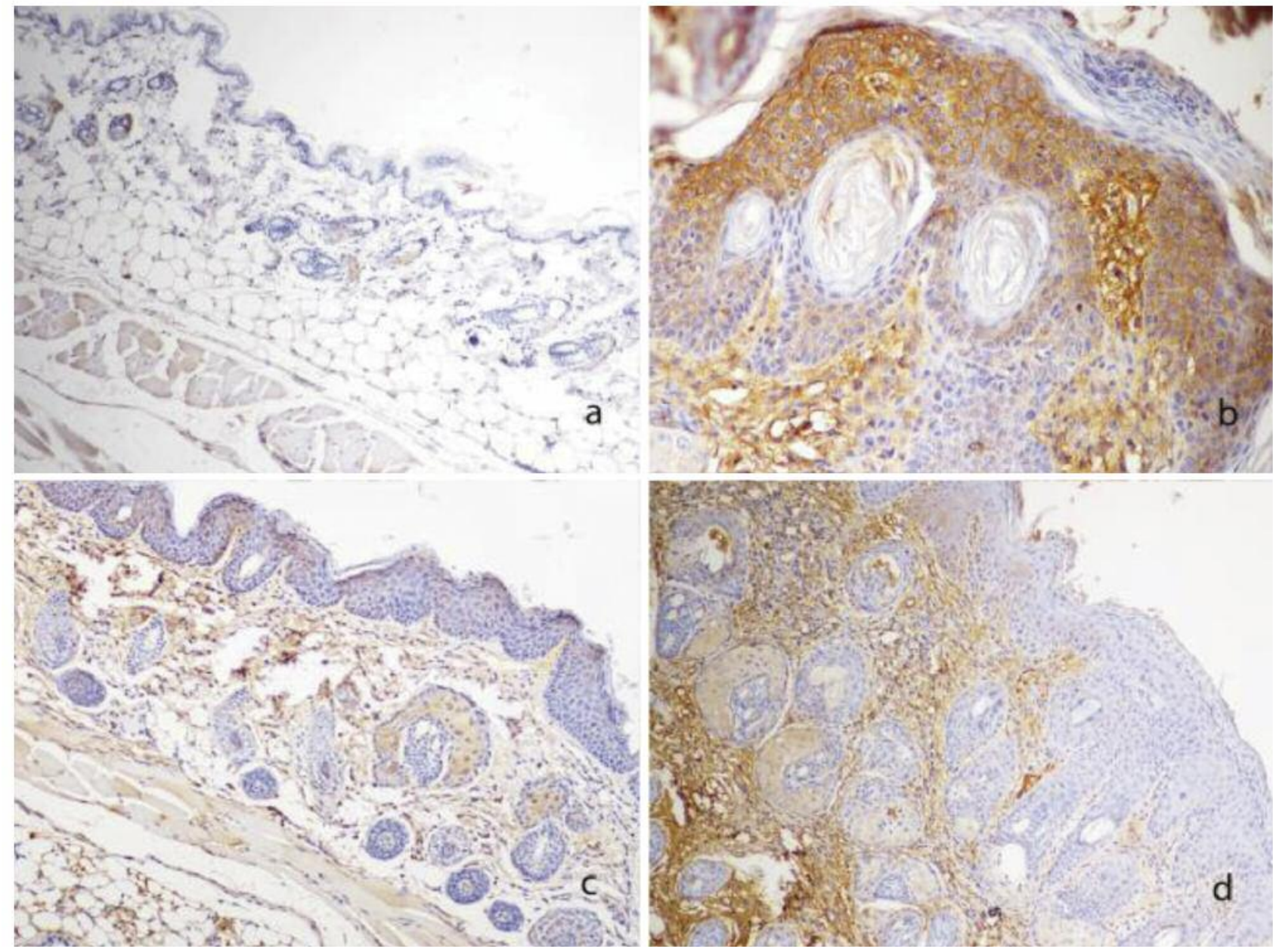

Figure 2. Cycle-oxygenase-2 (COX2) immunoexpression in experimental mice, diaminobenzidine-Mayer's hematoxylin staining. a: Wild-type mouse (group 1). COX2 expression limited to scattered dermal leukocytes, $\times 40 . b$ : Untreated HPV16-transgenic mouse (group 2). Intense diffuse dermal and epidermal COX2 immunoexpression, $\times 100 . c$ : Rutin-treated HPV16-transgenic mouse (group 3). Light to moderate, multifocal COX2 immunoexpression in the dermis and epidermis, x40. d: Curcumin-treated HPV16-transgenic mouse (group 4). Light, multifocal COX2 immunoexpression epidermis, but intense diffuse immunoexpression in the dermis, $\times 40$. 
Table I. Leukocyte counts on normal skin and lesions from K14HPV16 mice. Data are the mean $\pm S D$ (mean rank).

\begin{tabular}{lcccccc}
\hline Group & Macrophages & Neutrophils & Lymphocytes & Mast cells & Plasma cells & Total leukocytes \\
\hline $1: \mathrm{HPV}^{-/-}(\mathrm{n}=12)$ & $0.7 \pm 0.5\left(6.5^{\mathrm{a}}\right)$ & $0.2 \pm 0.4\left(6.5^{\mathrm{a}}\right)$ & $0.5 \pm 0.7\left(7.1^{\mathrm{a}}\right)$ & $0.9 \pm 0.7\left(6.5^{\mathrm{a}}\right)$ & $0.2 \pm 0.4(15.1)$ & $2.3 \pm 0.8\left(6.5^{\mathrm{a}}\right)$ \\
2: $\mathrm{HPV}^{+/-}(\mathrm{n}=8)$ & $6.6 \pm 1.2(35.9)$ & $5.8 \pm 2.3(31.6)$ & $2.9 \pm 1.1(24.25)$ & $6.4 \pm 1.2(35.6)$ & $1.0 \pm 1.1(26.4)$ & $21.6 \pm 3.6(34.75)$ \\
$3: \mathrm{HPV}^{+/-}+$rutin $(\mathrm{n}=12)$ & $4.8 \pm 1.3(24.75)$ & $4.3 \pm 1.6(25.5)$ & $3.7 \pm 1.4(29.6)$ & $5.1 \pm 1.3(27.2)$ & $0.8 \pm 0.9(24.7)$ & $17.8 \pm 3.4(24.8)$ \\
$4: \mathrm{HPV}^{+/-}+$curcumin $(\mathrm{n}=12)$ & $5.5 \pm 1.7(28.7)$ & $5.2 \pm 1.9(30.6)$ & $3.9 \pm 1.6(30.8)$ & $5.0 \pm 1.4(26.7)$ & $1.0 \pm 1.0(26.6)$ & $19.5 \pm 3.3(29.4)$ \\
\hline
\end{tabular}

aMean rank statistically different from groups 2,3 and $4(p<0.05)$.

Table II. Immunostaining scores for cyclo-oxygenase-2 on normal skin and skin lesions from K14HPV16 mice for each experimental group. Data are the mean $\pm S D$ (mean rank).

\begin{tabular}{|c|c|c|c|c|c|c|}
\hline Group & Extension & Dermis intensity & Score & Extension & Epidermis intensity & Score \\
\hline 1: $\mathrm{HPV}^{-/-}(\mathrm{n}=12)$ & $0.0 \pm 0.0\left(6.5^{\mathrm{a}}\right)$ & $0.0 \pm 0.0\left(6.5^{\mathrm{a}}\right)$ & $0.0 \pm 0.0\left(6.5^{\mathrm{a}}\right)$ & $0.0 \pm 0.0\left(6.5^{\mathrm{a}}\right)$ & $0.0 \pm 0.0\left(6.5^{\mathrm{a}}\right)$ & $0.0 \pm 0.0\left(6.5^{\mathrm{a}}\right)$ \\
\hline 2: $\mathrm{HPV}^{+/-}(\mathrm{n}=8)$ & $3.8 \pm 0.5(31.25)$ & $3.0 \pm 0.0(32.5)$ & $11.3 \pm 1.4(34.75)$ & $3.5 \pm 0.8(36.3)$ & $2.9 \pm 0.4(37.25)$ & $10.0 \pm 2.3\left(39.9^{\mathrm{b}}\right.$ \\
\hline 3: $\mathrm{HPV}^{+/-}+$rutin $(\mathrm{n}=12)$ & $3.1 \pm 0.7(22.7)$ & $2.6 \pm 0.5(25.8)$ & $7.8 \pm 1.9(21.5)$ & $2.7 \pm 0.9(28.5)$ & $1.8 \pm 0.7(23.8)$ & $4.5 \pm 1.2(24.5)$ \\
\hline 4: $\mathrm{HPV}^{+/-}+$curcumin $(\mathrm{n}=12)$ & $3.8 \pm 0.4(32.5)$ & $2.8 \pm 0.5(28.5)$ & $10.5 \pm 1.9(31.3)$ & $2.1 \pm 0.7(23.3)$ & $2.2 \pm 0.4(27.3)$ & $4.5 \pm 1.5(24.8)$ \\
\hline
\end{tabular}

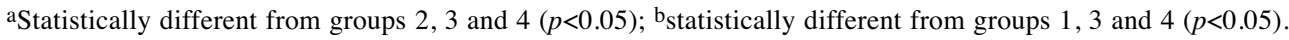

moderate to intense, diffuse $\mathrm{COX} 2$ immunoexpression in the epidermis (Table II), where it assumed a cytoplasmic or membrane-associated pattern. There was also intense, diffuse dermal COX2 immunoexpression (Figure 2b). The expression of COX2 was reduced by rutin administration in group 3 (Figure $2 c$ ) in the dermis (immunostaining score 7.83 versus 11.25 ) and epidermis (4.5 versus 10.0, $p<0.05)$ of transgenic mice. Curcumin (Figure 2d) also reduced the immunoexpression of COX2 in the epidermis ( 4.5 score, $p<0.05)$ but not on the dermis (10.5 score) of group 4 mice.

\section{Discussion}

Curcumin, isolated from the rhizomes of Curcuma longa, has long been used for nutritional and pharmaceutical purposes due to its anti-inflammatory and anti-neoplastic properties (17). Rutin, another polyphenolic compound, is a glycoside of the flavonoid quercetin, found in numerous edible plants and fruits. Rutin is one of quercetin's most common presentations and its digestion releases the sugar moiety, allowing the absorption of the quercetin aglycone (18). Curcumin inhibits the I kappa B kinase (IkK) complex, blocking I kappa B (IkB) phosphorylation and the nuclear translocation of NF-kB dimers, down-regulating COX2 (19), but not the expression of constitutive COX1 (20). Other polyphenols, such as quercetin, are also reported to down-regulate $\mathrm{COX} 2$ by inhibiting NF-kB signallng $(19,21)$. In many HPV-induced lesions, multistep carcinogenesis is accompanied by increasing inflammation, underpinned by $\mathrm{NF-kB}$, which is considered essential for tumor progression and metastasis (3).

Accordingly, in K14-HPV16 mice, the dysplastic stage coincides with enhanced sub-epidermal inflammation and angiogenesis $(22,23)$. In line with these findings, we observed significantly enhanced COX2 expression in skin samples from HPV16-transgenic animals compared with samples from wildtype ones (Figure 2). In transgenic mice, COX2 was overexpressed in the dermis and epidermis, accompanying the strong dermal inflammatory infiltrates observed in these animals. This agrees with the idea that HPV16 oncoproteins drive the expression of pro-inflammatory mediators to modulate the tumor microenvironment (2). Prolonged oral rutin administration effectively reduced dermal and epidermal expression of COX2, resulting in reduced leukocyte (mostly macrophages and neutrophils) infiltration (Table I), although this effect did not reach statistical significance. Curcumin was less effective, reducing epidermal, but not dermal COX2 expression and led to a more intense leukocyte infiltration, especially concerning macrophages and neutrophils. Although neither compound was able to reduce the incidence of dysplastic lesions, both showed interesting immunomodulatory properties in this model. This agrees with our previously published observations on this model (12), showing reduced systemic inflammation in mice treated with either curcumin or rutin. It is also in line with results by other research groups, which have shown quercetin to exert interesting antiinflammatory and anti-neoplastic properties (24). 
In the K14-HPV16 mouse model, curcumin seemed to exert a less potent anti-inflammatory effect, despite its known efficacy against some HPV-induced lesions (25), a discrepancy that may be explained by its poor pharmocokinetic properties (26). Taken together, the present results describe the expression pattern of $\mathrm{COX} 2$ in the K14HPV16 mouse model and help validate it for testing anti-inflammatory and immunoregulatory compounds. Both rutin and curcumin reduced COX2 expression in HPV16transgenic mice, but this was not sufficient to stop cancer progression. Future studies may take advantage of the antiinflammatory properties of these compounds by including them in combination therapies.

\section{Funding}

This study was funded by Liga Portuguesa Contra o Cancro, by the Research Center of the Portuguese Institute of Oncology of Porto (CIIPOP 37-2016), by project POCI-01-0145-FEDER-006939 (Laboratory for Process Engineering, Environment, Biotechnology and Energy), project POCI-01-0145-FEDER-006958 and UID/AGR/04033/2013, funded by FEDER funds through COMPETE2020 - Programa Operacional Competitividade e Internacionalização - and by national funds through the Fundação para a Ciência e a Tecnologia (FCT); Rui M. Gil da Costa was funded by grant number SFRH/BPD/85462/2012 from FCT, funded by the Portuguese Government and the Social European Fund.

\section{References}

1 Egawa N, Egawa K, Griffin $\mathrm{H}$ and Doorbar J: Human papillomaviruses; epithelial tropisms, and the development of neoplasia. Viruses 7: 3863-3890, 2015.

2 de Visser KE, Korets LV and Coussens LM: De novo carcinogenesis promoted by chronic inflammation is Blymphocyte dependent. Cancer Cell 7: 411-423, 2005.

3 Gil da Costa RM, Bastos MMSM, Medeiros R and Oliveira PA: The NFkB signalling pathway in papillomavirus-induced lesions: friend or foe? Anticancer Res 36: 2073-2083, 2016.

4 Subbaramaiah $\mathrm{K}$ and Dannenber AJ: Cyclooxigensa-2 transcription is regulated by human papillomavirus 16 E6 and E7 oncoproteins: evidence of a corepressor/coactivator exchange. Cancer Res 67: 3976-3985, 2007.

5 Kim SH, Oh JM, No JH, Bang YJ, Juhnn YS and Song YS: Involvement of NF-kappaB and AP-1 in COX2 up-regulation by human papillomavirus 16 E5 oncoprotein. Carcinogenesis 30: 753-757, 2009.

6 Herfs M, Herman L, Hubert P, Minner F, Arafa M, Roncarati P, Henrotin Y, Boniver $\mathrm{J}$ and Delvenne P: High expression of PGE2 enzymatic pathways in cervical (pre)neoplastic lesions and functional consequences for antigen-presenting cells. Cancer Immunol Immunother 58: 603-614, 2009.

7 Santos C, Neto T, Ferreirinha P, Sousa H, Ribeiro J, Bastos MM, Faustino-Rocha AI, Oliveira PA, Medeiros R, Vilanova M and Gil da Costa RM: Celecoxib promotes degranulation of $\mathrm{CD}^{+} \mathrm{T}-$ cells in HPV-induced lesions of K14-HPV 16 transgenic mice. Life Sciences 157: 67-73, 2016
8 Divya CS and Pillai MR: Antitumor action of curcumin in human papillomavirus associated cells involves downregulation of viral oncogenes, prevention of NFKB and AP-1 translocation and modulation of apoptosis. Mol Carcinogenesis 45: 320-32, 2006.

9 Dang YP, Yuan XY, Tuan R, Li DG and Liu W: Curcumin improves the paclitaxel-induced apoptosis of HPV-positive human cervical cancer cells via the NF-kB-p53-caspase-3 pathway. Exp Ther Med 9: 1470-6, 2005.

10 Velázquez KT, Enos RT, Narsale AA, Puppa MJ, Davis JM, Murphy EA and Carson JA: Quercetin supplementation attenuates the progression of cancer cachexia in ApeMin/+ mice. J Nutr 144: 868-75, 2014.

11 Shadfar A, Couch ME, McKinney KA, Weinstein LJ, Yin X, Rodríguez JE, Guttridge DC and Willis M: Oral resveratrol therapy inhibits cancer-induced skeletal muscle and cardiac atrophy in vivo. Nutr Cancer 63: 749-62, 3011.

12 Gil da Costa RM, Aragão S, Moutinho M, Alvarado A, Carmo D, Casaca F, Silva S, Ribeiro J, Sousa H, Ferreira F, NogueiraFerreira F and Pires MJ, Colaço B, Medeiros R, Venâncio C, Oliveira MM, Bastos MMSM, Lopes C and Oliveira PA: HPV16 induces a wasting syndrome in transgenic mice: amelioration by dietary polyphenols via NFkB inhibition. Life Sciences 169: 11$19,2017$.

13 Arbeit JM, Münger K, Howley PM and Hanahan D: Progressive squamous epithelial neoplasia in K14-human papillomavirus type 16 transgenic mice. J Virol 68: 4358-68, 1994.

14 Paiva I, Gil da Costa RM, Ribeiro J, Sousa H, Bastos M, Faustino-Rocha A and Lopes C, Oliveira PA and Medeiros R: MicroRNA-21 expression and susceptibility to HPV-induced carcinogenesis - role of microenvironment in K14-HPV16 mice model. Life Sci 128: 8-14, 2015.

15 Paiva I, Gil da Costa RM, Ribeiro J, Sousa H, Bastos M, Faustino-Rocha A and Lopes C, Oliveira PA and Medeiros R: A role for microRNA-155 expression in microenvironment associated to HPV-induced carcinogenesis in K14-HPV16 transgenic mice. PLoS One 10: e0116868, 2015.

16 Carvalho MI, Pires I, Prada J, Raposos TP, Gregório H, Lobo L and Queiroga LF: High COX2 expression is associated with increased angiogenesis, proliferation and tumoural inflammatory infiltrate in canine malignant mammary tumours: a multivariate survival study. Vet Comp Oncol 15: 619-631, 2016.

17 Shehzad A, Wahid F and Lee YS: Curcumin in cancer chemoprevention: molecular targets, pharmocokinetics, bioavailability, and clinical trials. Arch Pharm Chem Life Sci 9: 489-499, 2010.

18 Erlund I, Kosonen T, Alfthan G, Mäenpää J, Perttunen K, Kenraali J, Parantainen J and Aro A: Pharmacokinetics of quercetin from quercetin aglycone and rutin in healthy volunteers. Eur J Clin Pharmacol 56: 545-53, 2000.

19 Surh YJ, Chun KS, Cha HH, Han SS, Keum YS, Park KK and Lee SS: Molecular mechanisms underlying chemopreventive activities of anti-inflammatory phytochemicals: down-regulation of COX.2 and iNOS through suppression of NF-kappa B activation. Mutat Res 480-481: 243-268, 2001.

20 Goel A, Boland CR and Chauhan DP: Specific inhibition of cyclooxygenase-2 (COX2) expression by dietary curcumin in HT-29 human colon cancer cells. Cancer Lett 172: 111-118, 2011. 
21 Ramyaa P, Krisnaswamy R and Padma W: Quercetin modulates OTA-induced oxidative stress and redox signalling in HepG2 cells - up-regulation of Nrf2 expression and down-regulation of NF-kB and COX2. Biochim Biophys Acta 1840: 681-692, 2014.

22 Smith-McCune K and Zhu YH, Hanahan D and Arbeit J: Crossspecies comparison of angiogenesis during the premalignant stages of squamous carcinogenesis in the human cervix and K14-HPV16 transgenic mice. Cancer Res 57: 1294-1300, 1997.

23 Santos C, Vilanova M, Medeiros R and Gil da Costa RM: HPV-transgenic mouse models: tools for studying the cancerassociated immune response. Virus Research 235: 49-57, 2017.
24 Russo M, Spaguolo C, Tedesco I, Bilotto S and Russo GL: The flavonoid quercetin in disease prevention and therapy: facts and fancies. Biochem Pharmacol 83: 6-15, 2012.

25 Mishra A and Das BC: Curcumin as anti-human papillomavirus and anti-cancer compounds. Future Oncol 11: 2487-2490, 2015.

26 Anand P, Kunnumakkara AB, Newman RA and Aggarwal BB: Bioavailability of curcumin: problems and promises. Mol Pharm 4: 807-818, 2007.

Received December 30, 2017

Revised January 25, 2018

Accepted January 30, 2018 